\title{
LEAF ARCHITECTURE, LIGNIFICATION, AND TENSILE STRENGTH DURING VEGETATIVE PHASE CHANGE IN ZEA MAYS
}

\author{
RonAlD A. BALSAMO, JosePh A.J. ORKWISZEWSKI \\ Department of Biology, Villanova University \\ 800 Lancaster Avenue, Villanova, PA 19085 USA \\ e-mail: ronald.balsamo@villanova.edu
}

(Received: January 21, 2008. Accepted: June 17, 2008)

\begin{abstract}
Background and Aims: Leaf morphology, anatomy, degree of lignification, and tensile strength were studied during vegetative phase change in an inbred line of Zea mays $(\mathrm{OH} 43 \times \mathrm{W} 23)$ to determine factors that influence mechanical properties during development.

Methods: Tensometer, light microscopy, histochemistry.

Key results: Mature leaf length increased linearly with plant development, peaked at leaves 7 and 8 (corresponding to the onset of the adult phase) and then declined. Leaf width was stable for leaves 1 through 3, increased to leaf 7, remained stable to leaf 10, and then declined through leaf 13. Lamina thickness was highest for leaf 1 and decreased throughout development. Leaf failure load to width ratio and failure load to thickness ratio increased with development suggesting that changes in leaf morphology during development do not entirely account for increases in failure load. Histochemical analyses revealed that leaf tensile strength correlates with percent lignification and the onset of anatomical adult features at various developmental stages.

Conclusions: These data demonstrate that in Zea mays lignification of the midrib parenchyma and epidermis may be directly correlated with increased tensile strength associated with phase change from juvenility to adulthood. Failure load and resultant tensile strength values are primarily determined by the percent tissue lignification and the appearance of leaf architectural characters that are associated with the transition from the juvenile to the adult phase. Increased mechanical stability that occurs during the phase transition from juvenility to adulthood may signify a fundamental change in strategy for an individual plant from rapid growth (survival) to reproduction.
\end{abstract}

KEY WORDS: leaf architecture, leaf biomechanics, lignification, vegetative development, Zea mays.

\section{INTRODUCTION}

The evolutionary pressures dictating leaf morphology for a specific taxon tends towards the maximization of photosynthetic capacity within a given microhabitat within a particular ecosystem or biome. To fully understand the achievement of this goal, various other selective pressures must be taken into account. The most obvious are those features that will compensate for gravity, the conservation of water (thus allowing for survival of the individual under seasonal extremes of humidity and rainfall), and incident herbivory. During plant development, these features are particularly relevant as the evolutionary goal shifts from survival to reproduction and the successful passing on of potentially favorable genes. All of these ancillary extant conditions can be addressed by the utilization of techniques that investigate the gross biomechanical properties of le-

Non-standard abbreviations:

T - tip; MT - midtip; MB - midbase; B - base of laminas aves, as mechanical stability during development, the optimization of water relations, and reduction in the incidence of herbivory may be influenced by architectural, anatomical, and chemical characteristics (Balsamo et al. 2003, 2005) and play a central role in the ability of any individual plant to survive to adulthood and successfully reproduce.

Leaves of most plants display morphological and physiological changes during development (Poethig 1988). In many species these changes are gradual and difficult to discern. However, certain grasses, including Zea mays, sequentially develop distinct leaf morphologies during embryonic, juvenile vegetative, adult vegetative, and adult reproductive phases of development (Poethig 1988; Lawson and Poethig 1995). Each stage of development has unique phytomers that exhibit different anatomical features. In Zea mays there are also one to three transitional leaves between the juvenile and adult vegetative stage (Bongard-Pierce et al. 1996; Orkwiszewski and Poethig 2000). Juvenile leaves are characterized by the presence of epicuticular wax, the absence of trichomes, lack of lignins in epidermal cell 
walls and spherical epidermal cells, while adult leaves have no epicuticular wax, have trichomes, stain positive for lignins in the cell walls of epidermal cells, and have rectangular-shaped epidermal cells (Poethig 1988; Lawson and Poethig 1995; Sylvester et al. 2001). Transitional leaves have distinct regions where their anatomy and morphology exhibit either juvenile or adult vegetative anatomical characters (Orkwiszewski and Poethig 2000).

Studies on the mechanical properties of grass leaves have elucidated much useful information such as the relationship of leaf tensile strength and stem flexibility to livestock palatability (O'Reagain 1993), digestibility (De Sousa et al. 1982), resistance to trampling (Sun and Liddle 1993), and more recently, drought tolerance (Balsamo et al. 2003, 2005). Although over 40 years ago Kneebone (1960) suggested that the stage of leaf development had an important impact on leaf tensile properties, this has not, to our knowledge, been systematically investigated for species displaying a clear developmental sequence along the stem such as that exhibited by Zea mays (Lawson and Poethig 1995).

Studies investigating palatability and trampling resistance to livestock for several forage grasses have reported positive correlations between leaf tensile strength and the de-

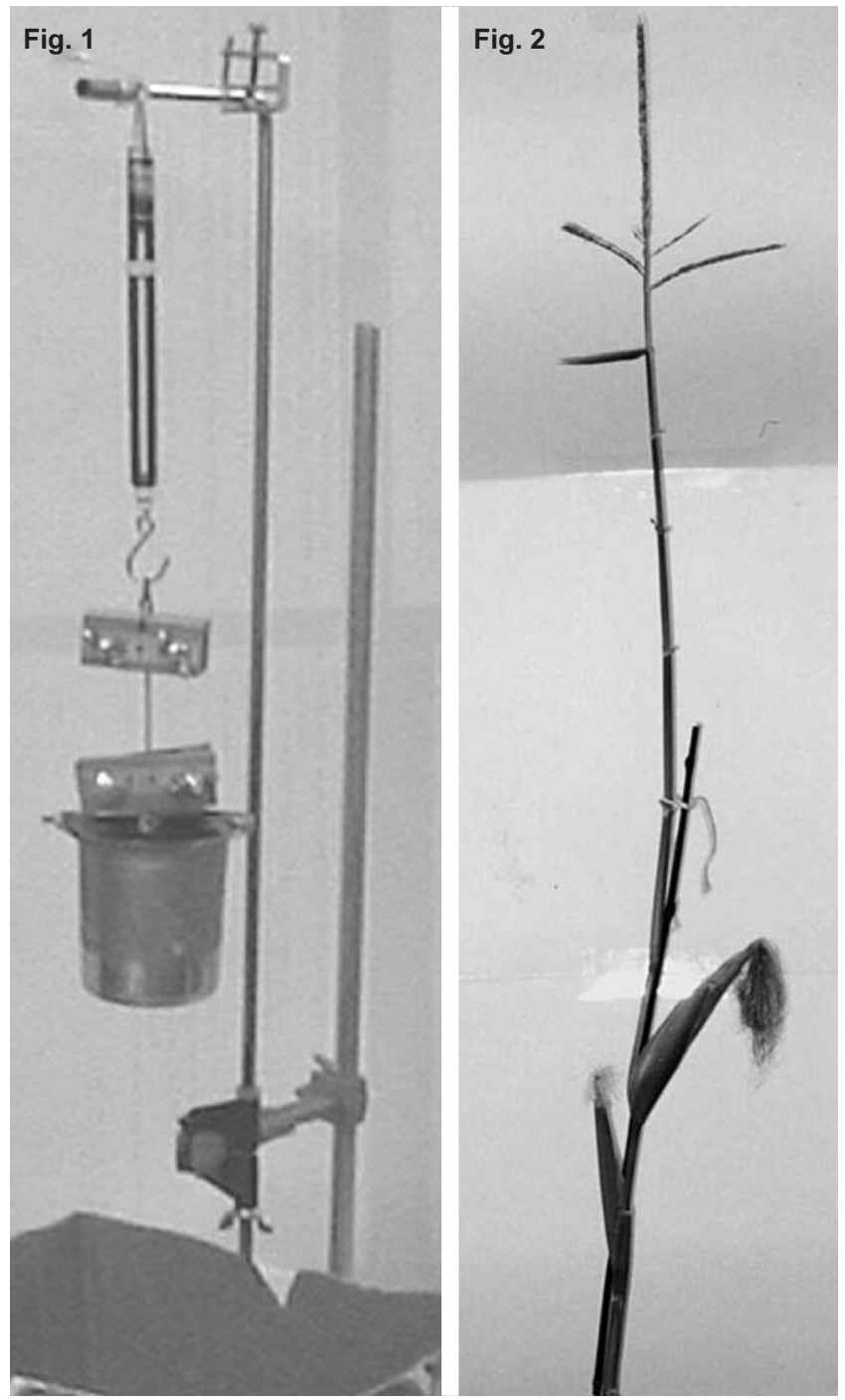

Figs 1 and 2. Fig. 1. Leaf tensometer used to measure lamina failure load. Fig. 2. Mature OH43 plant at end of study after leaves were sequentially removed when fully-expanded and demonstrating successful tassel and ear production. gree of lignification (De Sousa et al. 1982, Sun and Liddle 1993). Examining several species of South African forage grasses, Theron and Booysen (1968) suggested that the degree of lignification is the most important factor in the determination of tensile strength in immature grass leaves. Employing Lolium perenne as a model system, Vincent (1982) recognized the importance of the vascular system in leaf mechanical stability and its role as the major determinant of tensile strength in grasses. He estimated that up to $90 \%$ of the overall tensile properties of a grass leaf are determined by vascular tissues. More recent studies by Greenberg et al. (1989) also using L. perenne suggest that epidermal cells may also play a significant load bearing role in grass leaves, especially towards the tip where the size of the midrib, the vascular bundles, and the overall leaf thickness are generally diminished.

For Zea mays, there are clear qualitative and quantitative trends that correlate with phase transition from juvenile vegetative to adult vegetative leaves (Poethig 1988; Lawson and Poethig 1995; Bongard-Pierce et al. 1996; Sylvester et al. 2001). Intervenal distance decreases in the juvenile phase, increases in transitional leaves, and finally stabilizes in adult leaves. Similarly vascular area (the area occupied by vascular tissues as a percentage of total leaf cross sectional area) decreases in juvenile leaves, and subsequently increases throughout the transition and adult leaves. Cuticle thickness increases throughout the life cycle, while leaf thickness, the overall epidermal cell area (as measured in lamina cross sections), and the vascular bundle sheath area decreases (Bongard-Pierce et al. 1996). Given these data, the relationship of leaf phase (and concomitant characteristic anatomy and morphology) to tensile strength is likely to be complex. We hypothesize that in Zea mays, tensile strength will increase with the onset of lignification in leaf tissue and that the degree of lignification correlates closely with developmental phase change from juvenile to the adult vegetative state.

\section{MATERIAL AND METHODS}

\section{Plant material}

Seeds from an inbred line of Zea mays, OH43 $\times$ W23 (courtesy of R.S. Poethig, University of Pennsylvania, Philadelphia, Pennsylvania USA) were sown in Metromix 200 planting media: sand: peat moss $(50: 25: 25)$ in flats in the greenhouse $\left(25 / 20^{\circ} \mathrm{C}\right.$ day/night, $70 \%$ relative humidity). Upon germination and establishment seedlings were transferred to $15 \mathrm{~cm}$ pots and finally, $50 \mathrm{~cm}$ pots after the emergence of the first adult leaves. Individual leaves were collected for processing upon achieving maximum growth.

\section{Leaf morphology, anatomy, and tensile strength measurements}

Tensometers were constructed using 10, 25, and 50 Newton (N) Pesola scales (Baar, Switzerland), a mounting bracket, clamps, weather stripping, a metal beaker, and duct tape (Fig. 1). Whole laminas were excised from plants and cut into 200-300 mm segments. Leaf segments were designated as follows: Basal 200-300 mm (B), Mid-basal next 200-300 mm (MB), Midtip next 200-300 mm (MT), 200$300 \mathrm{~mm}$ closest to tip (T). Not all leaves had four segments because length varied according to their position along the 
stem and their developmental stage. The width and thickness of each segment was measured midway down its length to $\pm 0.01 \mathrm{~mm}$ using a ProMax digital caliper (Fowler instruments, Boston, Massachusetts USA). Laminas were clamped in place and lead pellets $(1.5 \mathrm{~g}$ each) were added to the beaker until catastrophic failure of the leaf blade occurred. Tensile strength was calculated by dividing the failure load by the cross-sectional area of the lamina $\left(\mathrm{N} / \mathrm{mm}^{2}=\mathrm{MPa}\right)$. This technique precluded the collection of the toughness and modulus of elasticity values due to the lack of continuous output monitoring. As a control 25 plants were grown along with the experimental plants but no leaf tissue was collected. Fully expanded leaves of adult leaf 8 showed no significant differences in width, length, or thickness between experimental plants (where leaves were being sequentially excised) and controls ( $P>0.05)$. In addition, all experimental plants successfully produced tassels and ears (Fig. 2).

\section{Histochemical analysis of leaves}

Seven plants were grown from seed under the conditions described above. At the proper developmental stage fully expanded leaves were excised, segmented, and processed for light microscopy as follows: Leaf 1 was excised from plants 1, 2, and 3. Leaf 2 was excised from plants 2, 3, and 4 etc. on through leaf 13 . One to two $\mathrm{cm}$ pieces from each cross section along the lamina $(\mathrm{B}, \mathrm{MB}, \mathrm{MT}, \mathrm{T})$ were excised and fixed in formaldehyde: acetic acid: ethanol: water (5:5:50 v/v, balance of distilled water) overnight. Samples were then dehydrated through an ethanol series and transferred into Citrisolv (Fisher Scientific, Pittsburgh, Pennsylvania USA) and slowly infiltrated with liquid paraffin over 3 d. Subsequently, samples were poured into plastic holders and solidified over crushed ice. Sections (4-6 $\mu \mathrm{m})$ were cut on a rotary microtome (American Optical, Buffalo, New York USA), de-paraffinized in Citrisolv, rehydrated in an ethanol series, and stained with Toluidine Blue $\mathrm{O}$, a metachromatic stain for cellulose and lignin (Lawson and Poethig 1995; Orkwiszewski and Poethig 2000). Photographs were obtained using an Olympus BX60 light microscope with an Olympus SC $35 \mathrm{~mm}$ camera (Tokyo, Japan). Determination of \% lignified cells was carried out using an Axiocam digital camera (Zeiss, Hallbergmoos, Germany) and AxioVision 2.05 software (Carl Zeiss Vision $\mathrm{GmbH}$, Hallbergmoos, Germany). Axiovision software was used to measure the total cross-sectional area of the photographed leaf sections and the cross-sectional area of lignin per leaf section. Four images per leaf were analyzed (leaves 1, 3, 5, 6, 7, 8 and 10).

\section{Statistical analysis}

Statistical tests were performed using the JumpStat 4 program software. Two-way ANOVA was followed by pairwise t-tests for different segments along the length of the lamina for each leaf age as well as between analogous segments for leaves of different ages.

\section{RESULTS}

\section{Leaf morphology and anatomy}

Leaf length (Fig. 3) and width (Fig. 4) increased with development from the first juvenile leaf through the transition
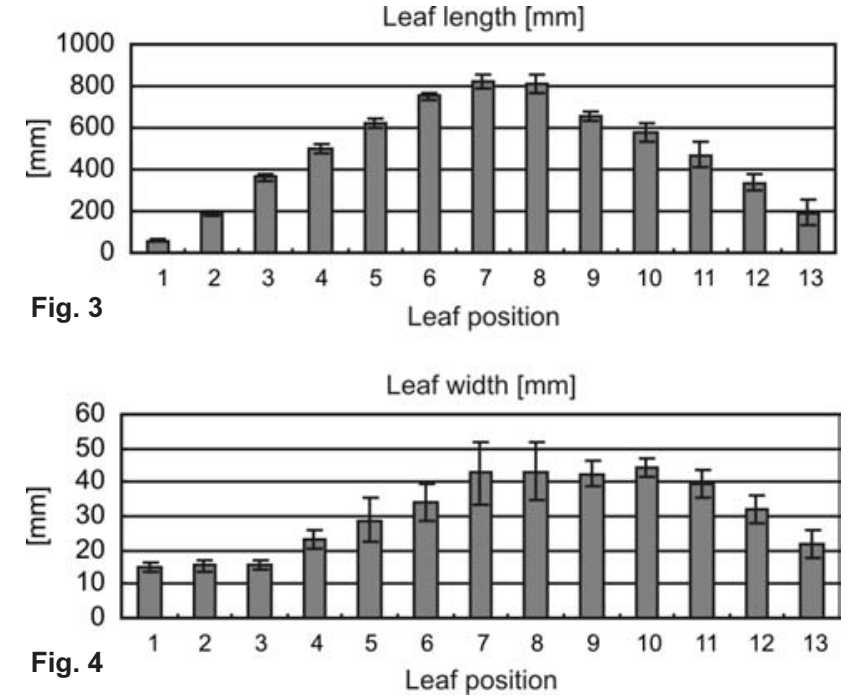

Figs 3 and 4. Fig. 3. Lamina length vs. position along stalk (bottom to top). Fig. 4. Lamina width vs. position along stalk (bottom to top). Bars indicate means $\pm \mathrm{SD} ; \mathrm{N}=7$.

to adult leaves and decreased thereafter until the formation of the tassel. Leaves 1-3 differed the most from each other and from adult leaves for length measurements $(\mathrm{P}<0.01)$. Similarly width was significantly less in juvenile leaves $(\mathrm{P}<0.05)$ when compared to adult leaves. In contrast, leaf thickness decreased with development in all measured segments along the lamina (Figs 5-7). Juvenile leaves were significantly different (thicker) than adult leaves along the length of the lamina $(\mathrm{P}<0.05)$. Juvenile leaves had roughly spherical epidermal cells with a thin cuticle and lignification (as evidenced by visual staining) was restricted to vessel elements and epithem cells of larger veins (Figs 8, 9,
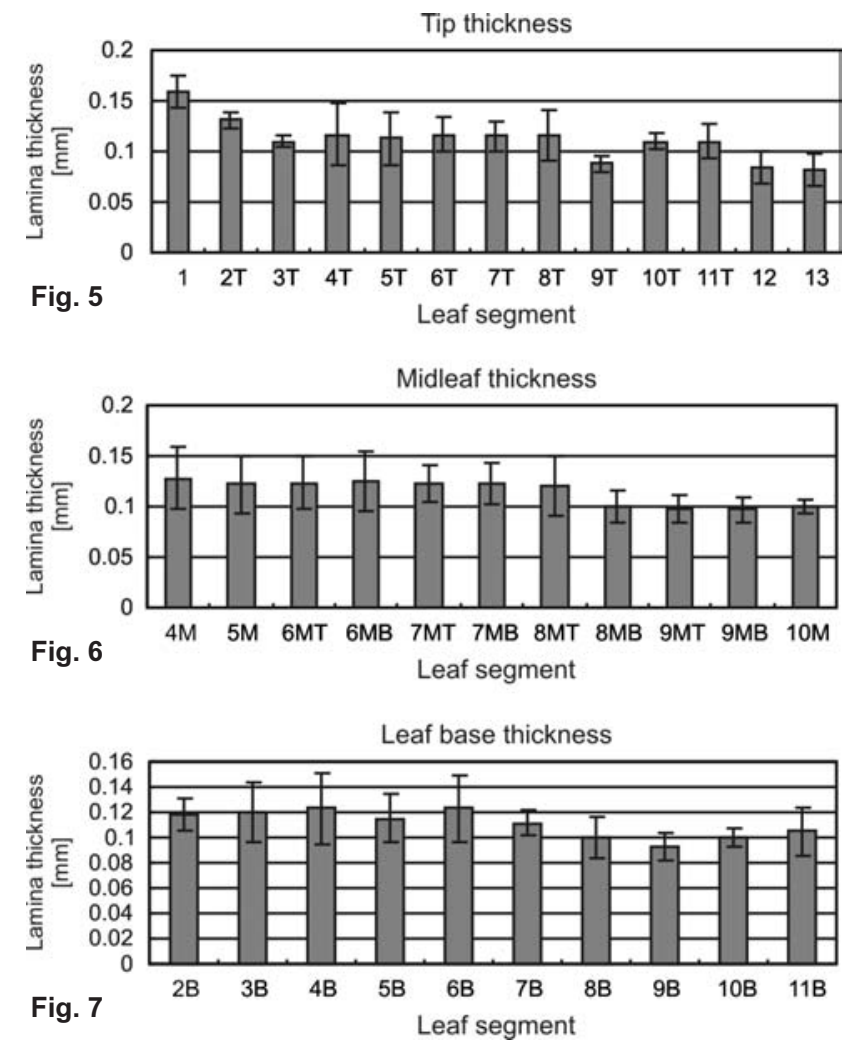

Figs 5-7. Fig. 5. Lamina thickness at tips. Fig. 6. Lamina thickness at midleaf. Fig. 7. Lamina thickness at base. Bars indicate means $\pm S D ; N=7$. 


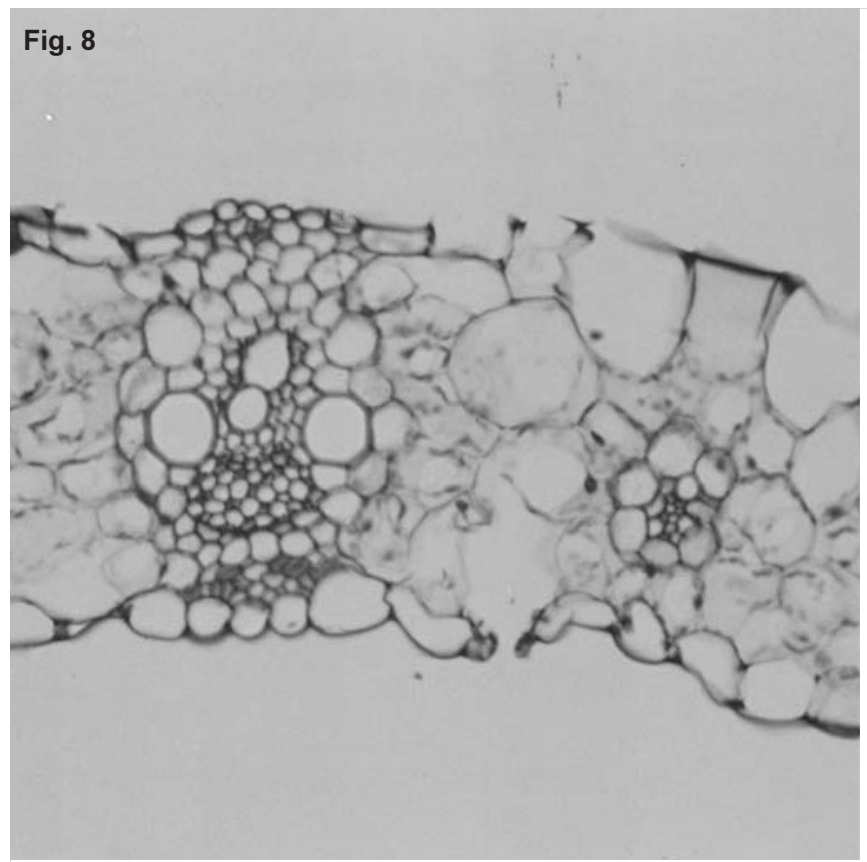

Fig. 10

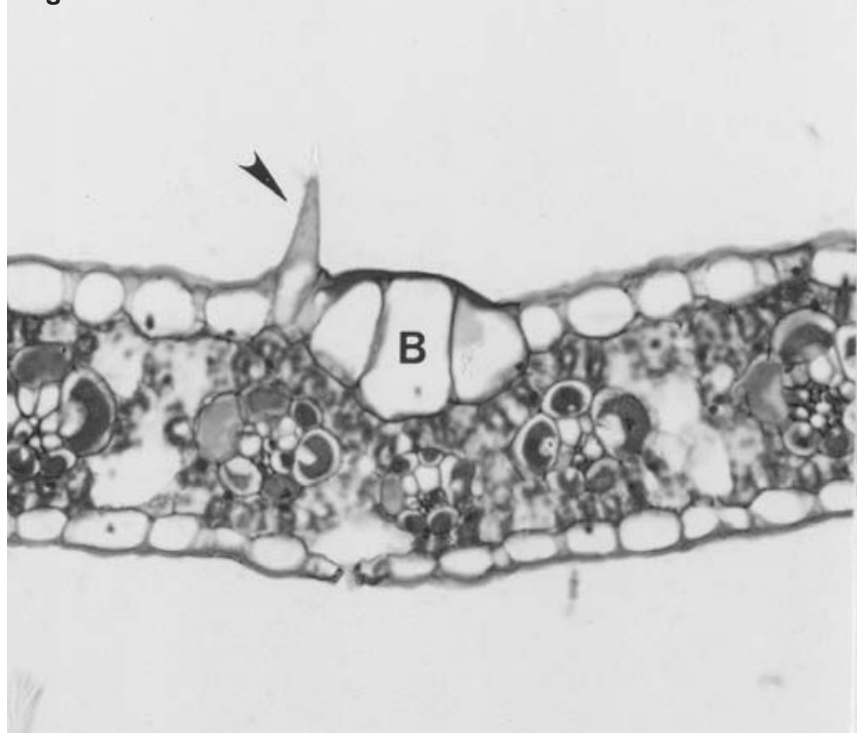

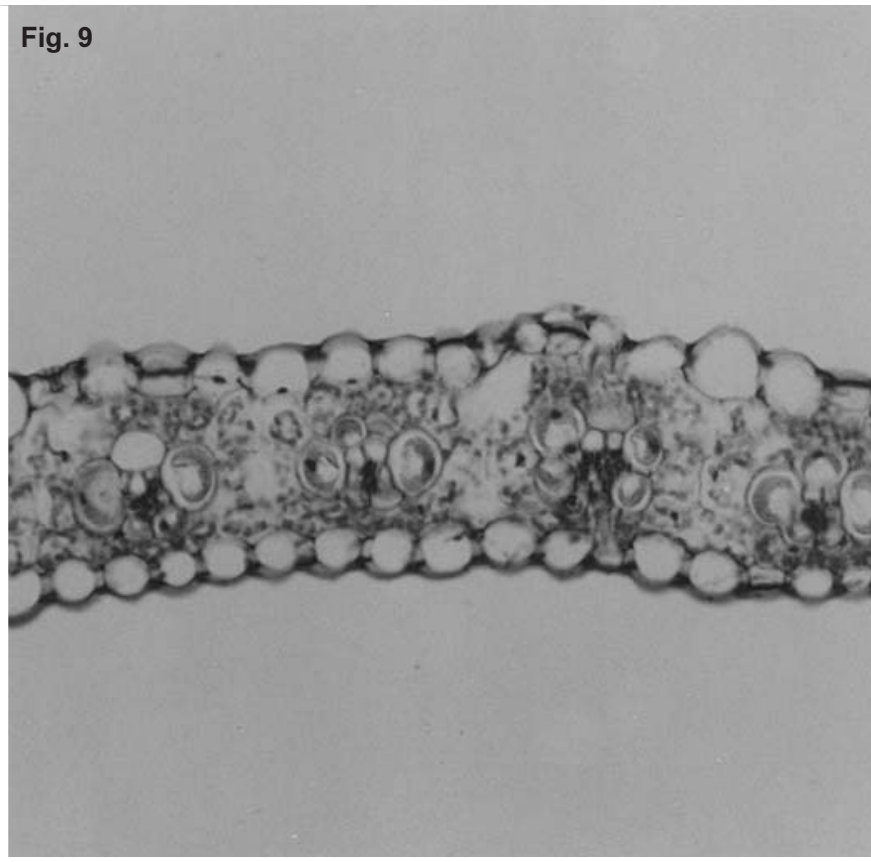

Fig. 11

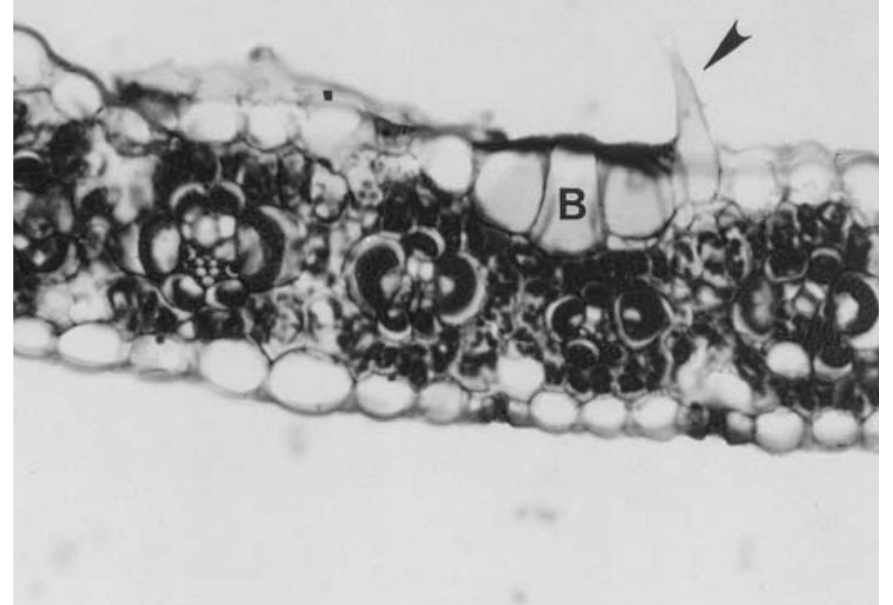

Figs 8-11. Fig. 8. Lamina cross sections at tip of leaves. Leaf 1. Fig. 9. Leaf 3. Fig. 10. Leaf 8. Fig. 11. Leaf 10. B - bulliform cells, arrows indicate trichomes; purple stain - unlignified tissues; light blue stain - lignified tissues. All micrographs photographed at 200x.

12, 13). There were no bulliform cells or epidermal hairs on either the adaxial or abaxial epidermal surfaces. Adult leaves had rectangular epidermal cells and thick cuticles (Figs 10, 11, 16, 17) and bulliform cells and trichomes were clearly evident. Transitional leaves exhibited juvenile characteristics at the tip (Fig. 18) and adult characteristics in the middle of the lamina and at the base (Figs 19-21). Leaf tips were the last part of the leaves to change from juvenile to vegetative adult with tips not showing fully adult morphological and histochemical features (in terms of lignification) until leaf 10 (Fig. 11).

\section{Histochemistry}

The metachromatic stain Toluidine blue O stains non-lignified cell walls of maize purple and lignified cell walls blue/green (Lawson and Poethig 1995; Orkwiszewski and Poethig 2000). Juvenile leaves did not exhibit lignification in any tissues except for the secondary walls of the xylem in the vascular bundles (Figs $8,9,12,13$ ) and some of the parenchyma cells in the midrib at the base of leaf 5 (Figs 14 and 15). Transition leaves 6 and 7 showed a gradient of lignification from the tip (least) to the base (most) in the midrib parenchyma cells, the epidermal cells, and the epithem of the vascular bundles (Figs 18-21). In leaves 8-13 there was an increase in lignification of the vascular bundles both at the tip (Figs 10 and 11) and the base (Figs 16 and 17). Interestingly, the cuticle of juvenile leaves and tissues (on transitional leaves) consistently stained purple while the cuticle of adult leaves and tissues stained blue, suggesting that chemical properties of the cuticle may also change with the developmental transition from juvenility to adulthood.

\section{Tensile properties}

Failure load increased with development. The failure load to width (Fig. 22) and failure load to thickness (Fig. 23) 

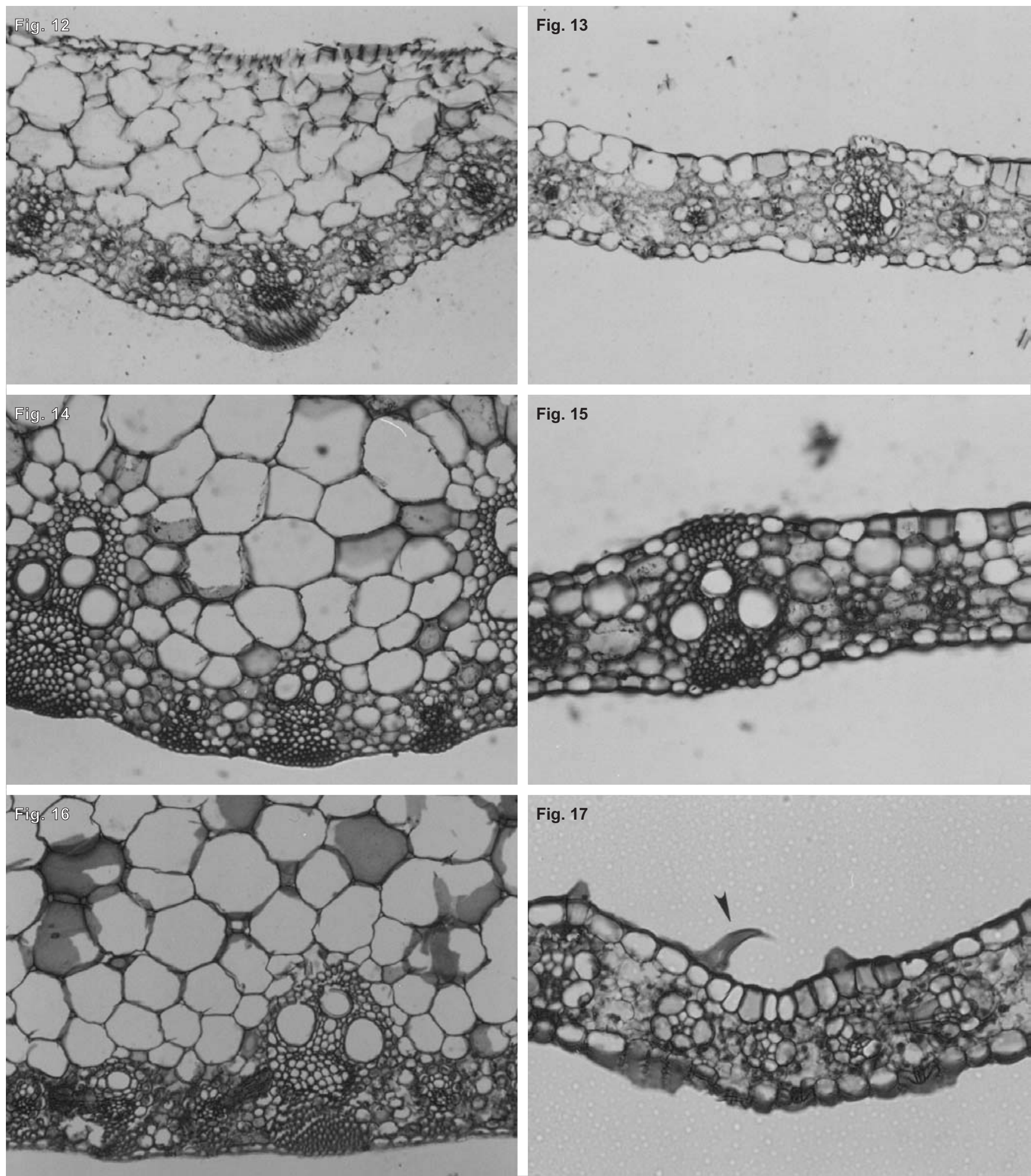

Figs 12-17. Leaf sections at base of leaves. Fig. 12. Midrib of leaf 3. Fig. 13. Lamina of leaf 3. Fig. 14. Midrib of leaf 5. Fig. 15. Lamina of leaf 5. Fig. 16. Midrib of leaf 8. Fig. 17. Lamina of leaf 8. Arrows indicate trichomes. Purple stain - unlignified tissues; light blue stain - lignified tissues. All micrographs photographed at 200x.

ratios increased from juvenile leaves through transition and adult leaves and then declined through leaves 10-13. Tensile strength values differed by stage of development as well as position along the lamina for leaves of a particular age and stage of development. Leaves 1 and 2 had significantly lower tensile strength values at the tips compared to all other leaves $(\mathrm{P}<0.05)$, and adult leaf tips had higher values overall than juvenile tips $(\mathrm{P}<0.05)$ (Fig. 24). Similarly, mid-tip segment tensile strength values increased from leaf 4 to leaf 10 (Fig. 25) showing a significant difference between juvenile and adult tissue $(\mathrm{P}<0.05)$. However, there was no significant difference in tensile strength between the midbase $(\mathrm{MB})$ transition $(6,7)$ segments and the adult leaf $(8,9) \mathrm{MB}$ segments (Fig. 26). The highest variability in tensile strength values were found in the leaf base samples (Fig. 27), most likely due to the confounding effects of 

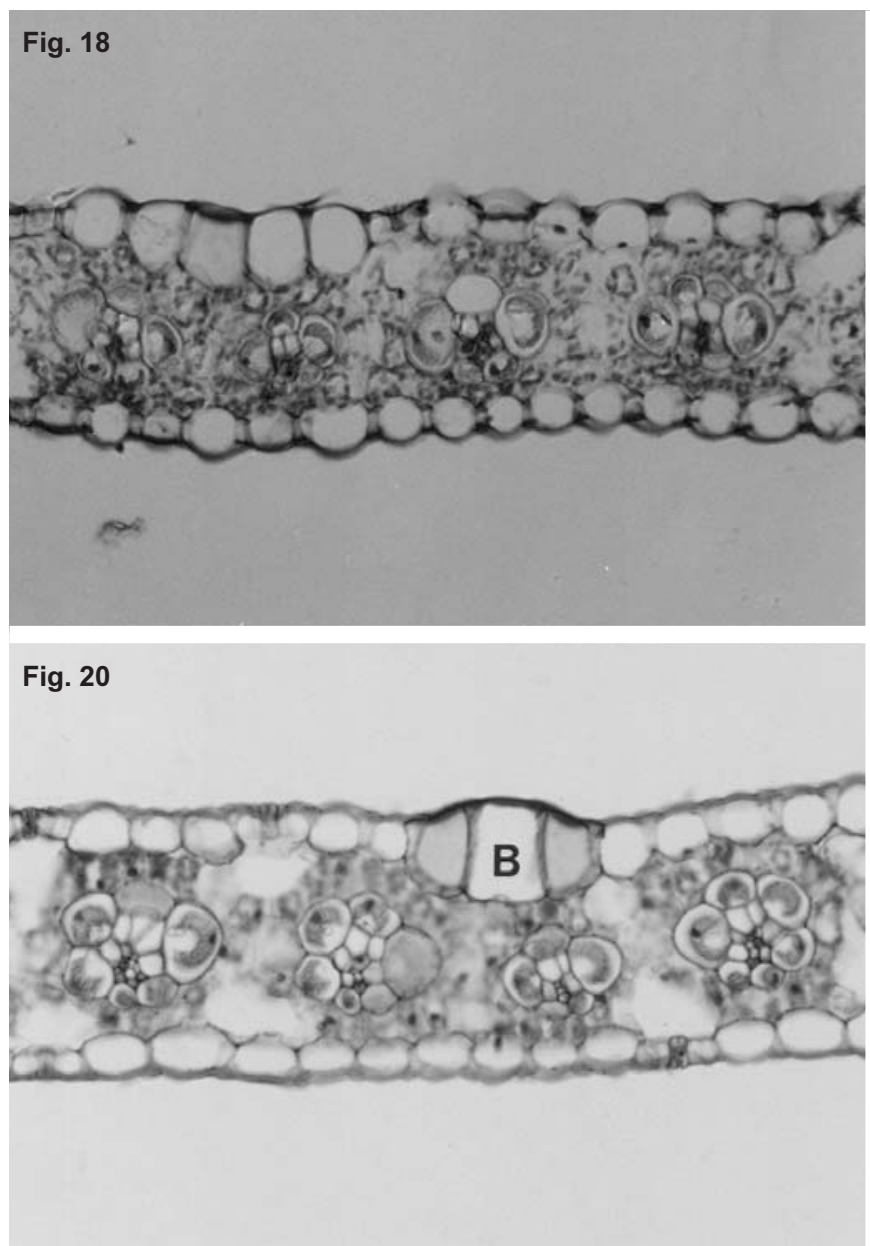

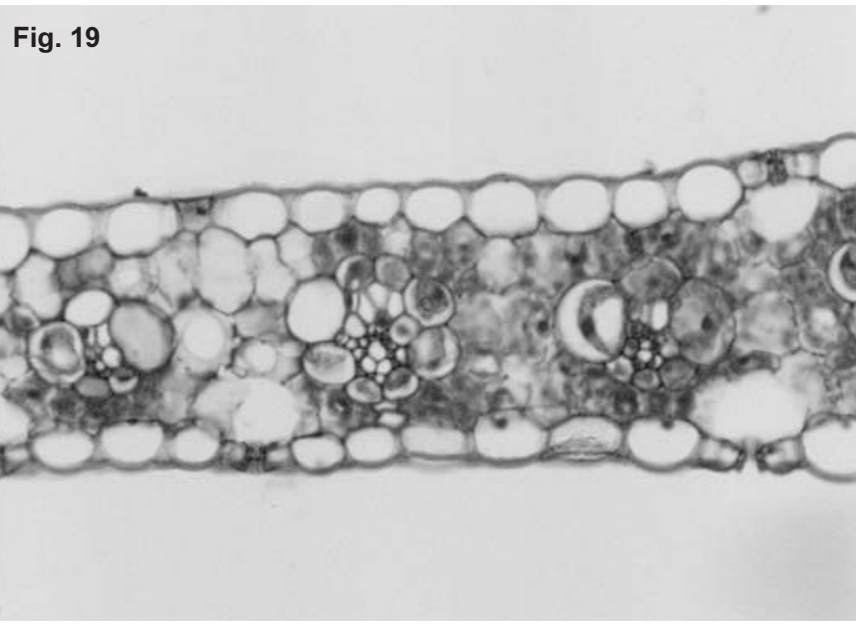

Fig. 21

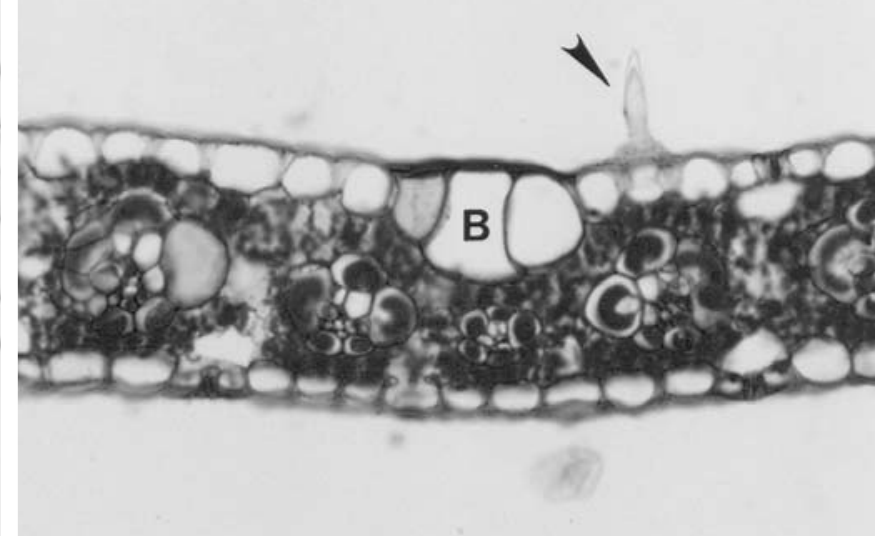

Figs 18-21. Lamina sections along a transitional leaf (6). Fig. 18. Tip. Fig. 19. Mid-tip. Fig. 20. Mid-base. Fig. 21. Base. B - bulliform cells, arrows indicate trichomes; purple stain - unlignified tissues; light blue stain - lignified tissues. All micrographs photographed at 200x.

a thickened midrib which complicated accurate measurements of lamina thickness, and was always the last part of the leaf base segment to fracture.

\section{Lignification}

The percent of lignified cells in leaf cross section increased with maturity (Fig. 28). Leaves 1, 3 and 5 exhibited increasing $\%$ lignified cells and were all significantly diffe-
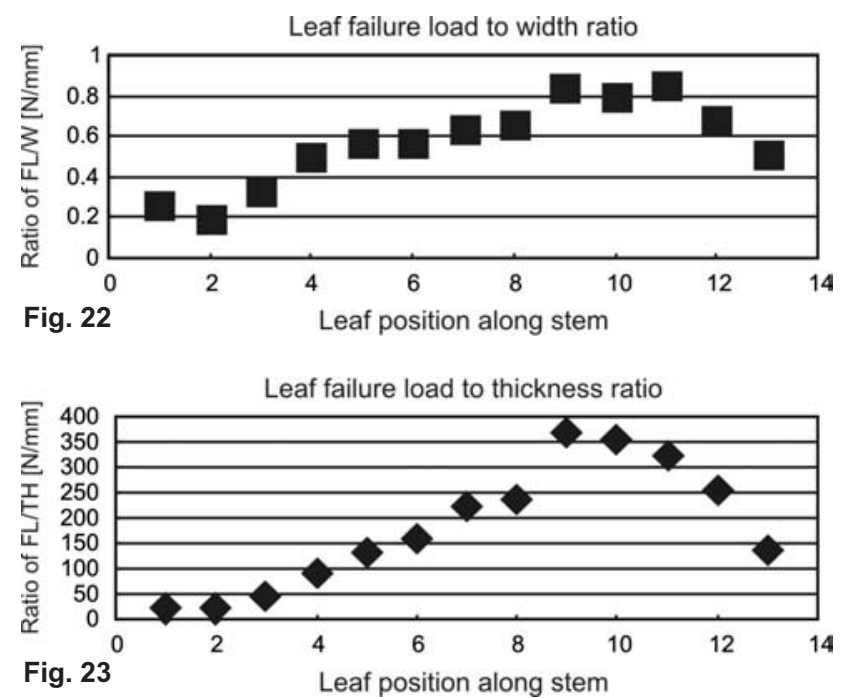

Figs 22 and 23. Fig. 22. Leaf failure load to width ratio for leaves 1-13. Fig. 23. Leaf failure load to thickness ratio for leaves $1-13 . \mathrm{N}=7$. rent from each other $(\mathrm{P}<0.05)$. Leaves 6,7 , and 8 were not significantly different from each other, but were significantly different from the juvenile leaves and leaf 10. Leaf 10 was significantly different from all other leaves.

\section{DISCUSSION}

These studies demonstrate that in Zea mays, the stage of leaf development has a strong influence on leaf tensile properties. The increase in failure load with development outpaces the increase in width and occurs despite a decrease in lamina thickness. Thus, changes in failure load and tensile strength (failure load corrected for leaf cross-sectional area) cannot be accounted for solely by changes in size and gross morphology. Leaf tensile strength also increased with development and was dependent upon position along the lamina. Our results demonstrating that leaf tensile strength decreases from the base to the tip of laminas in Z. mays is consistent with previous studies on other grasses (Kneebone 1960; Martens and Booysen 1968; Balsamo et al. 2005). A large part of the reduction in tensile strength in Z. mays from the base to the tip of the laminas in the current study may have been due to the reduction of the prominent midrib. Nevertheless, the increase in lignification of epidermal tissue most likely also plays a role, as evidenced by the increased tensile strength values of the adult leaf tips when compared to the juvenile leaf tips. Previous studies on the tensile properties of grasses have not been consistent. Early 

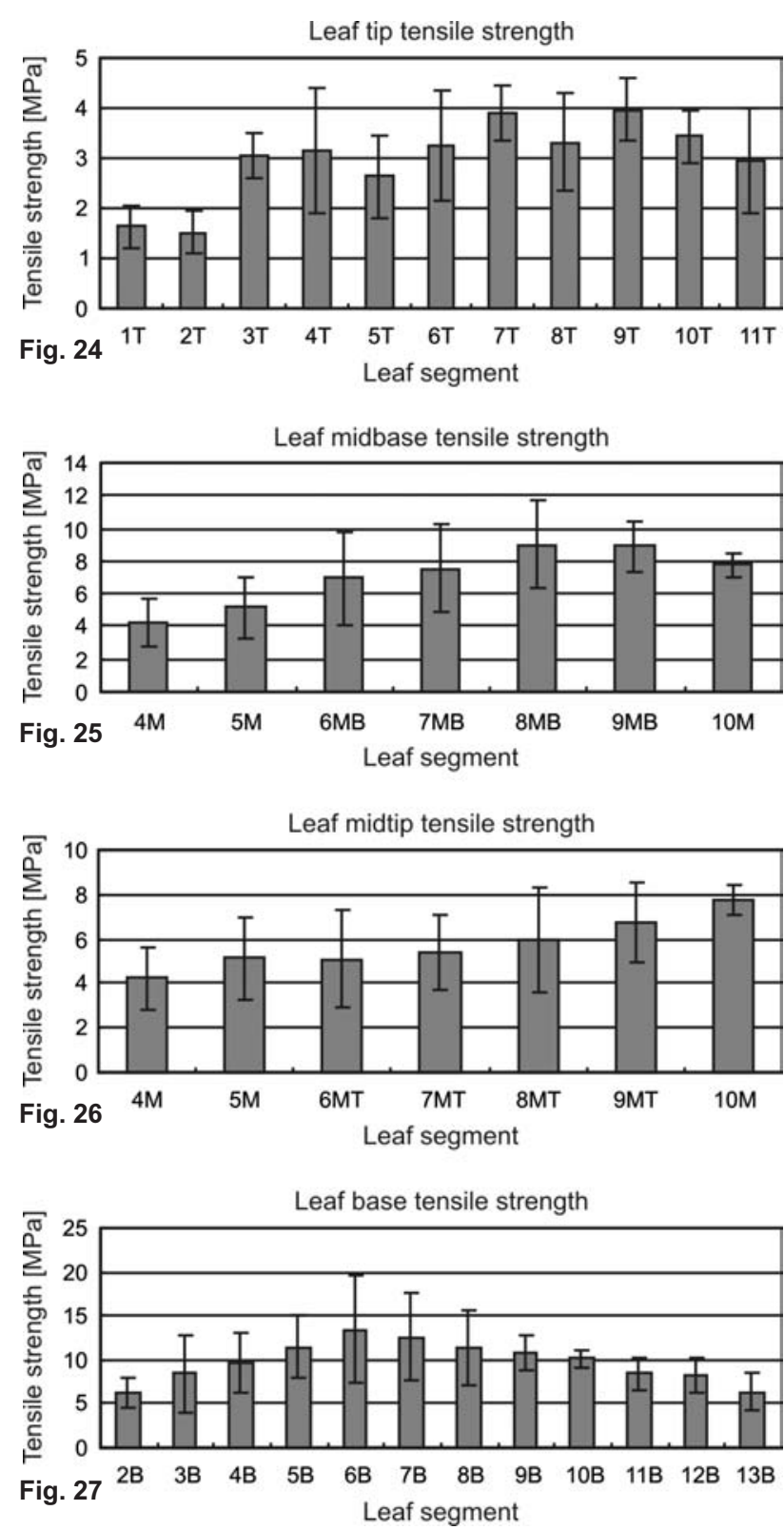

Figs 24-27. Leaf tensile strength values along the length of the lamina for leaves 1-13. Fig. 24. Tip. Fig. 25. Mid-tip. Fig. 26. Mid-base. Fig. 27. Base. Bars indicate means $\pm \mathrm{SD}$ and $\mathrm{N}=7$.

studies on forage grasses (Kneebone 1960; Martens and Booysen 1968) reported that breaking force (failure load) and tensile strength decreased from the base of the leaf to the tip. However, Greenberg et al. (1989) report that in Lolium perenne the fracture strength (failure load), stiffness (elastic modulus), and toughness (work to fracture) were higher at the tip than the base. These authors suggest that epidermal cells may play a significant role as a load-bearing component of the lamina for this species. Our results in Zea mays are consistent with earlier studies in that failure load and tensile strength consistently decreased from the base to the tip of the lamina. However, the tensile strengths of leaf tips in our study were higher in adult tissue than in juvenile tissue (Fig. 24), which may also support the observations of Greenberg et al. (1989). Indeed, when a prominent midrib is absent (like L. perenne or the tips of $Z$. ma$y s)$ lignification of the epidermal cells seems the most like-

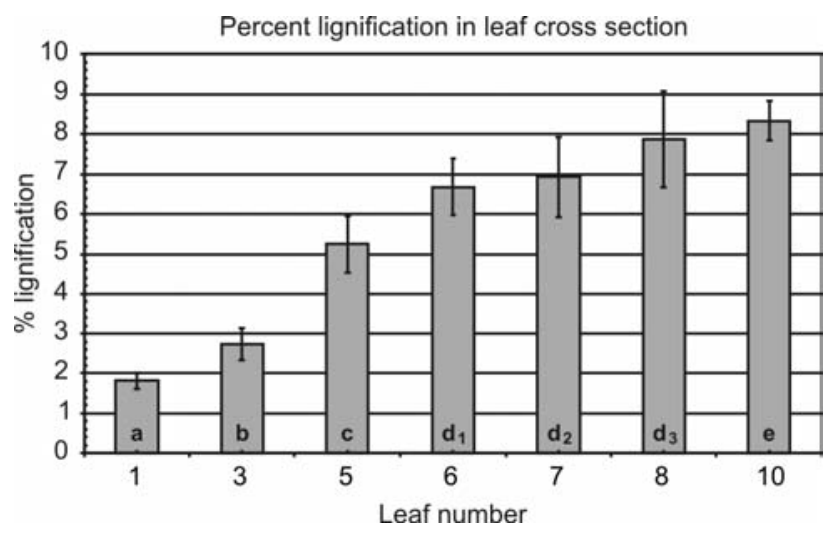

Fig. 28. Percent lignification of leaf cross sections vs. leaf number (counted from the base). Leaves 1, 3, and 5 show significantly increasing lignification and are different from each other and all other leaves $(\mathrm{P}<0.05)$ Leaves 6,7 , and 8 show increasing lignification but are not significantly different from each other $(\mathrm{P}>0.05)$. Leaf 10 is significantly different from leaves $1,3,5$, and $6(\mathrm{P}<0.05)$ and demonstrates the greatest degree of lignification. Different letters in histogram bars indicate significant differences $(\mathrm{P}<0.05)$ between samples. $\mathrm{N}=4$ per developmental stage.

ly explanation for increased tensile properties. Our observations agree with previous studies that described lignification of the cell walls of epidermal cells with the transition from the juvenile to the adult vegetative phase (Lawson and Poethig 1995; Orkwiszewski and Poethig 2000). We suggest that lignification of the cell walls of epidermal cells may be an important determinant in tensile strength for the tips of the leaves of Zea mays.

Little information is available with respect to the relationship of leaf tensile properties to development in grasses. Kneebone (1960) suggested that, along with growth stage, leaf age may be the most important factor causing variation in leaf tensile properties. Our results (increased tensile strength with phase change and increased \% lignification of leaf tissues) are consistent with studies on the leaves of Festuca arundinacea (De Sousa et al. 1982) which suggest that the amounts of cellulose and lignin in leaf samples have a significant impact on tensile properties. These studies indicate that phase transition between the juvenile and adult vegetative state in Zea mays correlates with increased tensile strength in the leaves. We suggest that this increase is primarily due to the progressive lignification of specific tissues in the lamina and that this relationship correlates closely with the transition from the juvenile to the adult vegetative stage of development. It is of interest to note that juvenile leaves tend to be ephemeral, dying off before tasseling while adult, lignified leaves survive throughout the season and are thus likely to be the main source of photosynthate for the developing tassels and ears. To our knowledge, this is the first report on the tensile properties in this species, and as such provides data that may be extrapolated to further developmental studies, such as the effects of different environmental influences on phase change, the use of inbred lines of genetic mutants to study changes in leaf physiological and mechanical (loadbearing) aspects with phase change, as well as studies correlating leaf water relations and responses to stresses such as drought, and mechanical injury to stability and drought tolerance. 


\section{ACKNOWLEDGEMENTS}

The Authors thank E.J. Gettings for assistance in the statistical analysis.

\section{LITERATURE CITED}

BALSAMO R.A., BAUER A.M., DAVIS S.D., RICE B.M. 2003. Leaf biomechanics morphology, and anatomy of the deciduous mesophyte Prunus serrulata (Rosaceae) and the evergreen sclerophyllous shrub Heteromeles arbutifolia (Rosaceae). Am. J. Bot. 90: 72-77.

BALSAMO R.A., VANDER WILLIGEN C., BOYKO W., FARRANT J. 2005. Anamolous leaf tensile properties during dehydration may help elucidate mechanisms of desiccation tolerance in Eragrostis nindensis. Physiol. Plant. 124: 336-342.

BONGARD-PIERCE D.K., EVANS M.M.S., POETHIG R.S. 1996. Heteroblastic features of leaf anatomy in maize and their genetic regulation. Int. J. Plant Sc. 157 (4): 331-340.

DESOUSA F.B., SLEPER D.A., BELYEA R.L., MATCHES A.G. 1982. Leaf tensile strength, "in vitro" digestibility, and fiber component relationships in tall fescue. Pesquisa Agropecuaria Brasiliera Brasilia 17 (10): 1497-1504.

GREENBERG A.R., MEHLING M. LEE, BOCK J.H. 1989. Tensile behaviour of grass. J. Mater. Sci. 24: 2549-2554.
KNEEBONE W.R. 1960. Tensile strength variations in leaves of weeping lovegrass (Eragrostis curvula (Schrad.) Nees.) and certain other grasses. Agr. J. 52: 539-542.

LAWSON E.J.R., POETHIG R.S. 1995. Shoot development in plants: time for a change. Trends in Genetics 11: 263-268.

MARTENS P.O., BOOYSEN P de V. 1968. A tensilmeter for the measurement of the tensile strength of grass leaf blades. Proc. Grassl. Soc. S. Afr. 3: 51-56.

O'REAGAIN P.J. 1993. Plant structure and the acceptability of different grasses to sheep. J. Range Manage. 46: 232-236.

ORKWISZEWSKI J.A.J., POETHIG R.S. 2000. Phase identity of the maize leaf is determined after leaf initiation. P.N.A.S., USA 97: 10631-10636.

POETHIG R.S. 1988. Heterochronic mutations affecting shoot development in maize. Genetics 119: 959-973.

SUN D., LIDDLE M.J. 1993. Trampling resistance, stem flexibility and leaf strength in nine Australian grasses and herbs. Bio. Conserv. 65: 35-41.

SYLVESTER A.W., PARKER-CLARK V., MURRAY G.A. 2001. Leaf shape and anatomy as indicators of phase change in the grasses: comparison of maize, rice, and bluegrass. Am. J. Bot. 88 (12): 2157-2167.

THERON E.P., BOOYSEN P. de V. 1968. The tensile properties of ten indigenous grasses. Proc. Grassl. Soc. S. Afr. 3: 57-61.

VINCENT J.F.V. 1982. The mechanical design of grass. J. Mater. Sci. 17: 856-860. 